UCRL-JC-130775

PREPRINT

\title{
Compact and Stable Multibeam Fiber Injector
}

\author{
Leland F. Collins
}

This paper was prepared for submittal to the Current Developments in Optical Design and Engineering VII

San Diego, CA

July 19-24, 1998

July 1998

This is a preprint of a paper intended for publication in a journal or proceedings. Since changes may be made before publication, this preprint is made available with the understanding that it will not be cited or reproduced without the permission of the author. 


\section{DISCLAIMER}

This document was prepared as an account of work sponsored by an agency of the United States Government. Neither the United States Government nor the University of California nor any of their employees, makes any warranty, express or implied, or assumes any legal liability or responsibility for the accuracy, completeness, or usefulness of any information, apparatus, product, or process disclosed, or represents that its use would not infringe privately owned rights Reference herein to any specific commercial product, process, or service by trade name, trademark, manufacturer, or otherwise, does not necessarily constitute or imply its endorsement, recommendation, or favoring by the United States Government or the University of California The views and opinions of authors expressed herein do not necessarily state or reflect those of the United States Government or the University of California, and shall not be used for advertising or product endorsement purposes 


\title{
Compact and Stable Multibeam Fiber Injector
}

\author{
Leland F. Collins \\ Lawrence Livermore National Laboratory, L-281, Livermore California 94556
}

\begin{abstract}
A compact and stable 20-beam injector was built for launching laser light into fibers for Fabry Perot velocity measurements of shock-driven surfaces. The fiber injector uses commercial mounts on mini-rails. Dielectric-coated beamsplitters provide accurate amplitude division. Minimal adjustments for stable operation arc permitted by the use of a real-time video-viewer. The video system includes a non-linear camera for CW alignment and a linearized camera with a frame grabber for pulsed measurement and analysis. All 20-injection points are displayed on a single monitor. Optical requirements are given for image relay and magnification. Stimulated Brillouin scattering limitations on high-power are quantified.
\end{abstract}

Keywords: Laser Doppler velocimetry, long-pulse YAG laser, amplitude division, thin-film beamsplitters, fiber-injection viewer, Stimulated Brillouin Scattering (SBS).

\section{Laser Doppler Velocimetry}

In laser-Doppler velocimetry, laser light suffers a frequency change as it reflects or scatters from a moving surface. Very large frequency shifts can be quantified using diffraction gratings or even prisms. Low frequency-shifts can be detected with heterodyne techniques and displayed on a spectrum analyzer. Velocities using scattered light from rapidly moving bodies moving in the range of $1-10 \mathrm{~mm} / \mu \mathrm{s}$ are best analyzed using interferometric methods. ${ }^{1}$ The figure below shows a schematic of a velocimeter using a Fabry Perot interferometer.

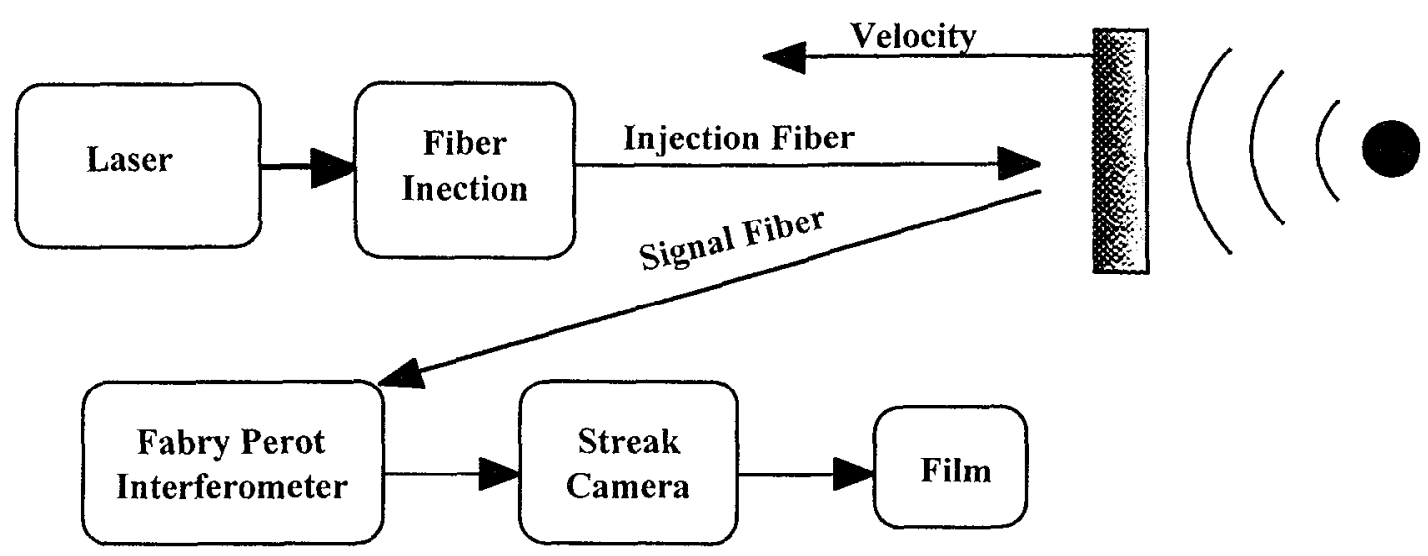

Figure 1: Laser-Doppler velocimeter schematic. Numerous beam collecting and shaping optics are not shown.

Light from a frequency-doubled pulsed-laser goes to an injection system where it is divided and injected into each of several different fibers. Fiber light then moves to an explosively driven surface. Diffusely reflected and Doppler shifted light from the surface is captured by a signal fiber and transported to the Fabry Perot interferometer. The Fabry converts frequency shifted light into a time-dependent interference pattern that is temporality moved across photographic film by a streaking camera so that the 
position of the interference fringes can be recorded. The output is a record of instantaneous surface-velocity versus time. Only the fiber injection portions of the velocimeter will be described in detail.

\section{Laser}

In our current generation of velocimeters, visible light is created by a long-pulse YAG laser system. This laser system uses a ring-based seed-laser with an extremely narrow linewidth, and four stages of amplification. It includes a Pockels cell for pulse-shape control, three Faraday isolators to keep stray light out of the seed laser, and three pinhole-filters for beam quality. The Joule-sized infrared output is converted to $532 \mathrm{~nm}$ light with $10 \%$ efficiency by a KTP doubling crystal. The pulse width is adjustable from 40 to 80 $\mu \mathrm{s}$. The laser system also contains a low-power continuous wave $532 \mathrm{~nm}$ laser for safely performing alignments. Laser output can be decreased with a polarization rotator coupled with a thin-film plate polarizer.

\section{Injection Invariant}

A key parameter in determining the nature of light injected into fibers is the optical invariant, which is given by ${ }^{2}$

$$
\Theta D_{0}=\frac{4 \mathrm{M}^{2} \lambda}{\pi}
$$

where $\Theta$ is the full-angle far-field beam divergence, $D_{0}$ is the waist-diameter and $\lambda$ is the wavelength. The parameter $\mathrm{M}$ is equal to unity for a laser operating in the fundamental Gaussian mode. Higher-order modes produce waist spot-sizes that are $\mathrm{M}$ times larger than do those of a fundamental Gaussian. Similarly, divergence measured far from the waist is larger than that of a Gaussian by the factor $M$. It is useful to lump the effect of all higher-order beam modes in the single parameter $M$.

For efficiency, light injected into optical fibers must be smaller than the fiber core and its angle of convergence must be smaller than the fiber Numerical Aperture, or NA. If the spot size is not smaller than the fiber core, then excess light is lost and it may even burn the fiber. Small-diameter fibers are desirable in Doppler-velocimetry because they can allow more accurate measurement. Low NA fibers are also desirable in velocimetry because they increase the measurement range while minimizing probe dimensions ${ }^{3}$. High values of the product of fiber NA and core-size are not useful in high-resolution Fabry Perot interferometry.

In our original laser specification, the $\mathrm{M}^{2}$ was to be so large that light would not launch efficiently into small, low NA fibers. To work around this, a wavefront divider was designed for fiber injection. In wavefront division, an enlarged laser beam is separated into smaller-sized segments using mirrors as dividers. Wavefront division can result in beams with a smaller optical invariant. In amplitude-division, portions of the entire beam wavefront are sampled using partially reflective beamsplitters. With amplitude division, the optical invariant is maintained for each of the beamlets. The long-pulsed lasers finally manufactured had near-Gaussian beam quality and so the amplitude-divider described here was built.

\section{Fiber Injectors}

The splitter and lens system injects light into twenty fibers. Fiber cores are usually $100 \mu \mathrm{m}$ and the

numerical apertures are 0.07 or 0.11 . Dielectric coaled beansplitters were used with reflective values of 20 , $25,33,50$ and $100 \%$, as shown below. 


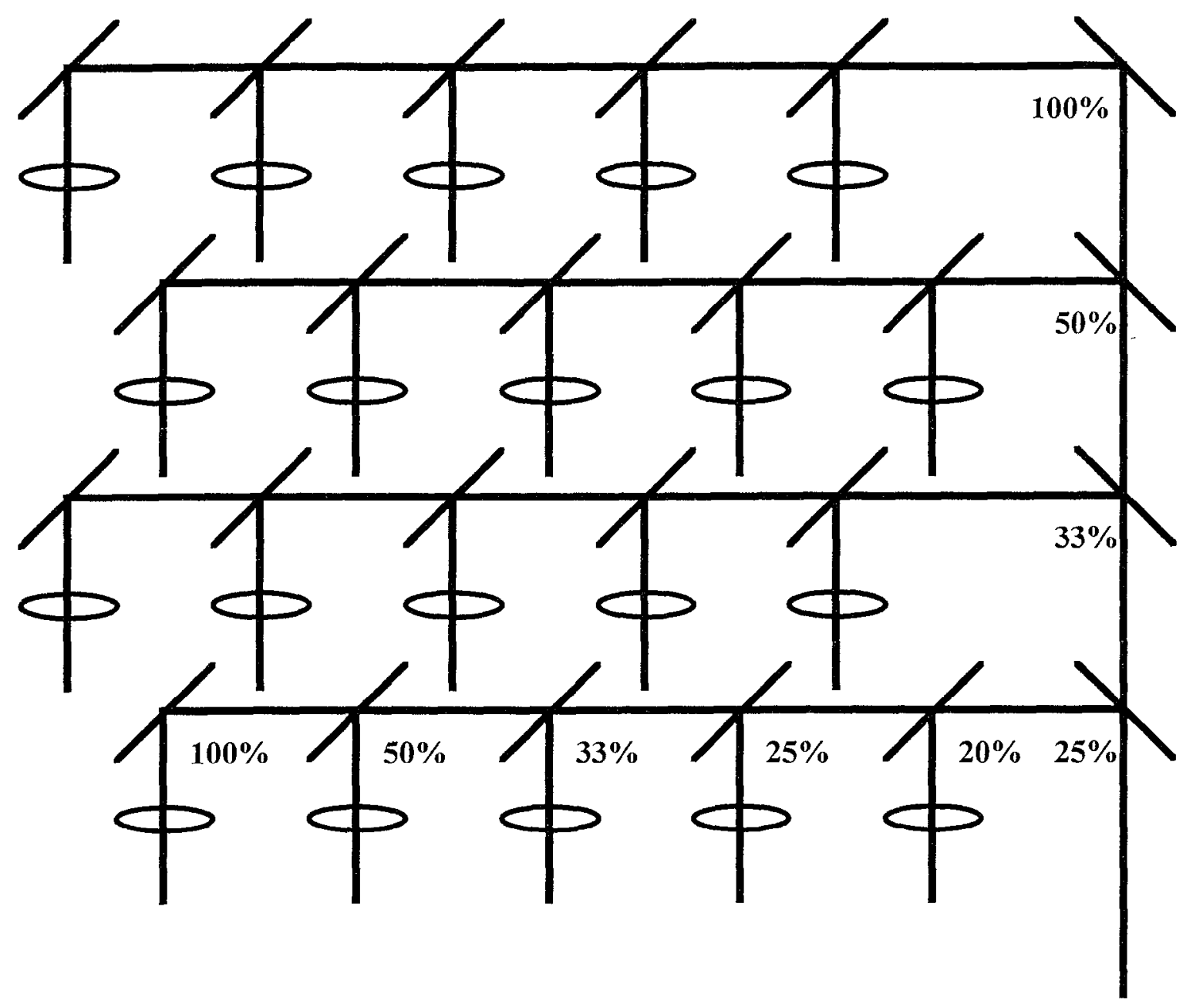

Figure 2: 20-Beam Injector

The splitters were designed for equal light of $5 \%$ to each fiber. Actual values are shown below.

$\begin{array}{llllll}\text { Leg \# } 4 & 4.9 & 4.4 & 4.3 & 4.5 & 4.2 \\ \text { Leg \# } 3 & 4.9 & 4.5 & 4.4 & 5.0 & 4.9 \\ \text { Leg \# 2 } & 5.2 & 4.8 & 4.9 & 5.5 & 5.2 \\ \text { Leg \# 1 } & 4.8 & 4.4 & 4.6 & 5.1 & 4.9\end{array}$

Table 1: Measured percentage of light delivered to each fiber of the 20-beam injector. The mean injection percentage is 4.8 , the standard deviation is 0.35 and the range is \pm 0.65 .

The injection lenses are $50 \mathrm{~mm}$ focal-length achromats. Input beam diameters at the lenses are about $3 \mathrm{~mm}$. Lens and splitter mounts and the lenses are catalogue items. The fiber holders and various mechanical interfaces were custom made. Most of the injector is mounted on mini-rails. The number of adjustments was minimized for system stability. Beams on the fiber faces are stable to $\pm 200 \mu$ radians for periods of weeks for room temperature changes below $2{ }^{\circ} \mathrm{C}$. Alignment between our present laser and injection platforms undergoes a thermal drift for larger temperature changes. 


\section{Fiber Viewer}

If an injected beam drifts off its fiber face, power is lost and the fiber may be damaged This section describes an optical viewer for precisely verifying injection alignment.

The Fresnel-reflected light from the fiber input face may go back through the injecting lens and reform into a magnified image of the injection spot. This image can be detected and viewed by placing a video camera in the plane where the magnified image occurs. Separating the reverse-traveling beam from the incident-laser light is done with an angled beamsplitter.

The fiber core can also be viewed, which provides a reference for centering the injected spot. Light injected into the fiber travels its length and a portion Fresnel reflects off the output end, retraverses the fiber, and exits the original injection end. If this light re-enters the injection lens, it forms a magnified image of the core. Light reflected from both ends of the fiber form images at the same location, as shown in Fig. 3 Both images can be amazingly crisp and laden with detail, although they are made of speckles.

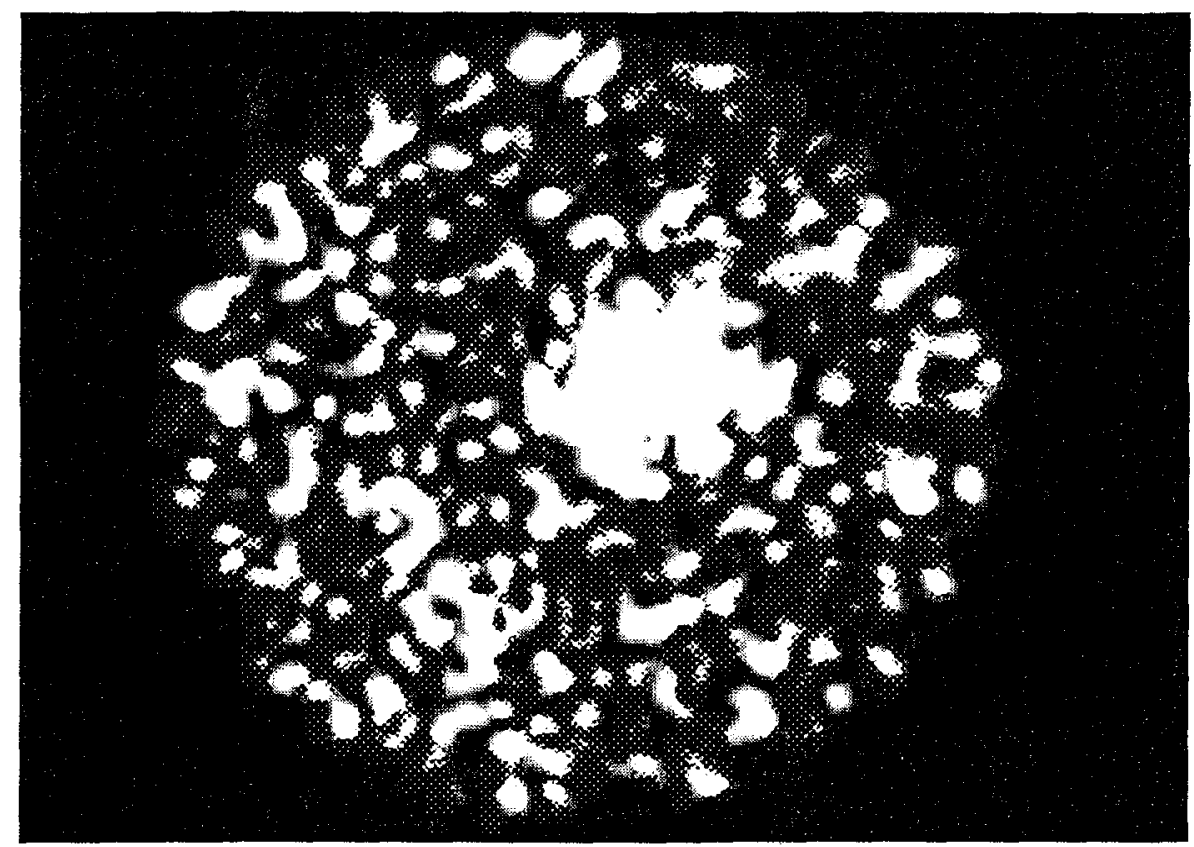

Figure 3: Video image of fiber reflections. The larger circle is the core image and the smaller off-center spot is the direct reflection from the input face.

A difficulty with fiber-face imaging is in obtaining the desired magnification when the beam waist is placed at the fiber face. If the injection-lens to fiber-face spacing is set so that the image magnification $\mathrm{m}$ is a particular value, then using the equation

$$
\frac{1}{\mathrm{Ra}_{\mathrm{a}}}+\frac{1}{\mathrm{~S}_{\mathrm{a}}}=\frac{1}{\mathrm{~F}_{\mathrm{a}}}
$$

yields the result that the lens-to-fiber spacing $R_{a}$ need be given by

$$
R_{a}=\left(I-\frac{1}{m}\right) F_{a},
$$

where $F_{a}$ is the injector-lens focal length and $S a$ is the lens-to-image spacing Here, both $R_{a}$ and $S_{a}$ are taken to be positive for real images. From eq. 2, setting a $50 \mathrm{~mm}$ lens for a factor of 25 magnification requires that $R_{\mathrm{a}}$ be $52 \mathrm{~mm}$. Magnifying a $100 \mu \mathrm{m}$ fiber core by 25 times projects a conveniently sized 2.5 mm image onto a $1 / 2$ " format Charge Coupled Device (CCD). 
The complication comes in that the minimum spot size of the incident beam may not occur at the fiber face when $R_{a}$ is set for the desired magnification. If the incident beam is $532 \mathrm{~nm}$ and it originates from a two-millimeter waist diameter located three meters upstream of the injection lens, then the focused beam has a waist some $502 \mathrm{~mm}$ downstream of the injection lens. Setting the fiber at $\mathrm{Ra}=50.2$ yields $m=-250$ and this places the image $12 \mathrm{~m}$ beyond the lens. If instead the fiber were set to make $\mathrm{Ra}=52$ for an $m=-25$ magnification, then the injected beam diameter at the fiber face would quadruple from its minimum value of $17 \mu \mathrm{m}$. These values may be verified using the Gaussian formulas quoted in the appendix.

This example illustrates that it may not be possible to simultaneously image the fiber face with an optimal magnification while minimizing the injected-beam waist. A solution is to use a reduction lens between the injection lens and the camera as shown in Fig. 4.

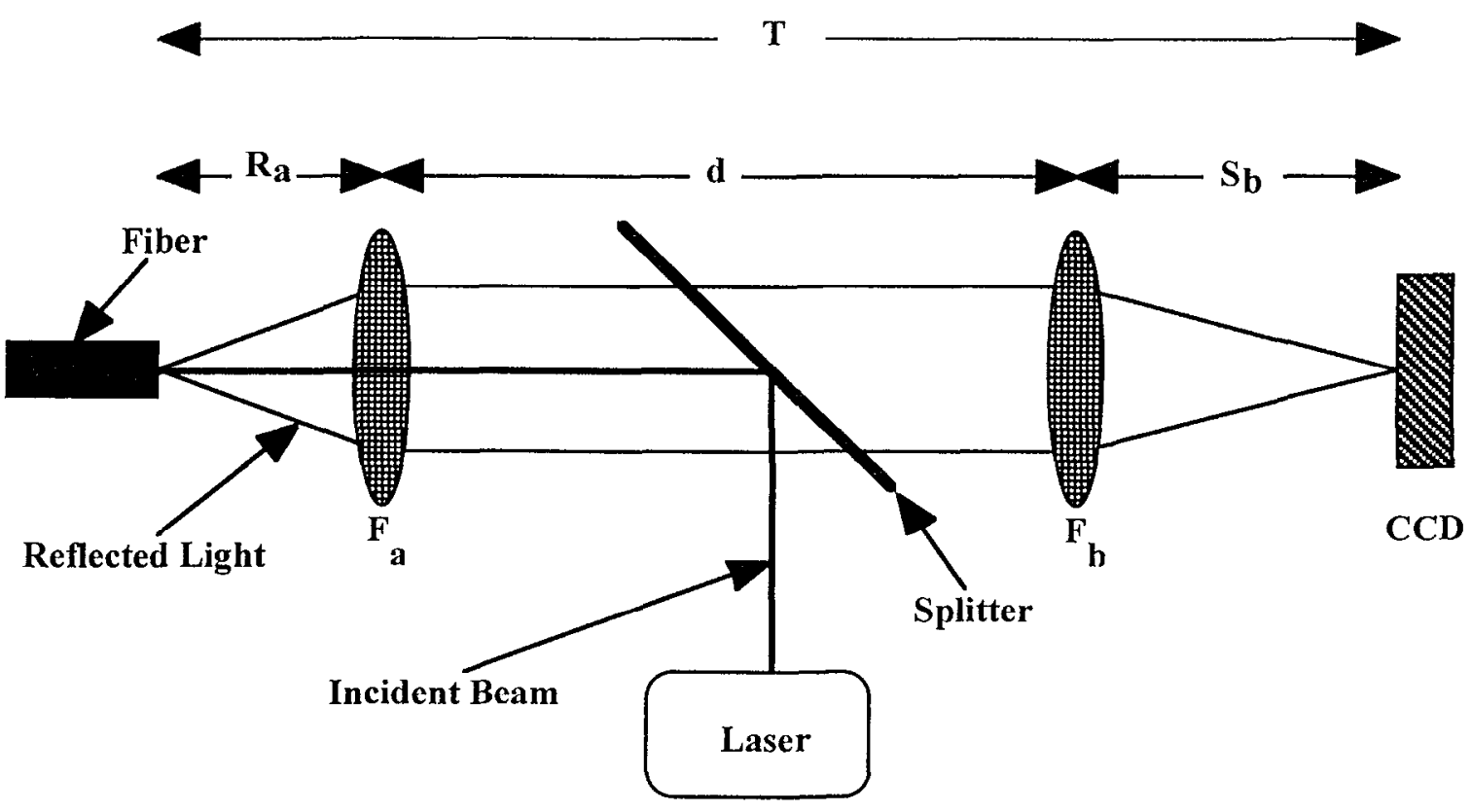

Figure 4: Fiber viewer showing incident laser beam reflecting from fiber, passing through injector lens, splitter and reduction lens, and then forming an image on the video CCD.

The distance $d$ between the injection lens and the reduction lens can be calculated with the following expression.

$$
d=\frac{R_{a}(m-1)+T}{\frac{m R_{a}}{F_{a}}-(m-1)}
$$

where $T$ is the pathlength between the fiber and the desired CCD location, $R_{a}$ is the required fiber to injection-lens spacing, and $m$ is the desired overall magnification. The optimal value for $R_{a}$ is determined by measuring the waist location and beamsize upstream of the injector, and then calculating $R_{a}$ with the Gaussian beam formulas listed in the Appendix. This procedure places the incident-beam waist on the fiber face. 
The spacing $\mathrm{Sb}$ between the reduction lens and the image (located at the CCD) is calculated with the following equation.

$$
\mathrm{S}_{b}=\mathrm{T}-\mathrm{d}-\mathrm{R}_{\mathrm{a}}
$$

Eq. 5

Finally, the focal length of the reduction lens $F_{b}$ may be calculated with this expression:

$$
F_{b}=\frac{d S_{b}}{d+m R_{a}+S_{b}}
$$

Convenient values for $T$ and $m$ are first chosen, then the spacing $d$ and focal length $\mathrm{Fb}_{b}$ are calculated with eqs. 4-6. Alternately, $T$ can be selected as a dependent variable by solving eq. 4 for $T$ in terms of $d$.

As a numerical example, values of $F_{a}=50, R_{a}=50.2, m=-25$ and $T=2000$ lead to $F_{h}=1310$ and $\mathrm{d}=770$.

It should be noted that if the fiber core is not perfectly parallel to the connector body, or if the fiber face is not perfectly perpendicular to the injected beam, then the directly reflected spot will move off to the side of light emanating from the core for non-image locations. Hence viewing of beams not located at the true core-image location can give an erroneous indication of spot centering.

Even though all the fiber images can appear on a single video display, there is a large difference between intensities. The light reflected from the front face is brighter than light reflected from the output face if the injected beam is smaller than the core. There is a difference in light levels between different fibers due to splitter efficiency. Tables 2 and 3 illustrate the fiber-to-fiber variance.

$\begin{array}{rrrrrr}\text { Leg \# 4 } & 1.7 & 1.7 & 1.7 & 1.7 & 1.7 \\ \text { Leg \# 3 } & 1.7 & 1.7 & 1.7 & 1.7 & 1.7 \\ \text { Leg \# } 2 & 1.7 & 1.7 & 1.7 & 1.7 & 1.7 \\ \text { Leg \# 1 } & 15 & 15 & 15 & 15 & 15\end{array}$

Table 2: Percentage of spectrally reflected s-polarized light reaching the camera. The total s-light from a single fiber is taken as $100 \%$. The splitter s-reflectivities are 20, 25, 33, 50 and $100 \%$.

$\begin{array}{rrrrrr}\text { Leg \# 4 } & 2.7 & 0.68 & 0.36 & 0.23 & 0.18 \\ \text { L.eg \# 3 } & 0.68 & 0.17 & 0.09 & 0.06 & 0.05 \\ \text { Leg \# 2 } & 0.36 & 0.09 & 0.05 & 0.03 & 0.02 \\ \text { Leg \# 1 } & 61 & 15 & 8.2 & 5.2 & 4.1\end{array}$

Table 3: Percentage of diffusely reflected p-polarized light reaching the camera. The total plight from a single fiber is taken as $100 \%$. The corresponding splitter p-reflectivities are 4.3 , $5.8,9.6,20$ and $100 \%$.

The specular light reflected from the injection surface is polarized, but light reflected from the far end of the fiber is usually depolarized. While return light from all the injection fibers can reach the same video camera, means must be provided to block all but one fiber reflection at a time. 


\section{Stimulated Brillouin Scattering}

Once the Brillouin power threshold is reached, coherent light injected into a fiber generates a forwardtraveling sound wave that in turn reflects incident light. Increasing the incident power beyond this threshold does not increase the throughput, as the "excess" power is returned out the injection end of the fiber. The Stimulated Brillouin Scattering (SBS) power threshold $\mathrm{Po}_{0}$ is given by

$$
\mathrm{P}_{0}=\frac{21 \mathrm{~A}}{\mathrm{gLeff}}
$$

where $A$ is the fiber-core area, Leff is the effective fiber length, the number 21 is an arbitrary constant used to designate large gain (some authors use the value 30 instead), and $\mathrm{g}$ is a material-dependent constant called the Brillouin gain. The effective fiber length Leff can be calculated from the following formula:

$$
\mathrm{L}_{\mathrm{cff}}=\frac{1-\exp (-\alpha \mathrm{L})}{\alpha}
$$

where $\alpha$ is the fiber's absorption coefficient and $\mathrm{L}$ is its length.

For polarization-preserving communication fiber and wavelength, $\mathrm{g}$ is approximately equal to $5 \times 10^{-11}$ $\mathrm{m} / \mathrm{W}$. The SBS threshold increases by about a factor of $3 / 2$ for unpolarized light $\left(\mathrm{g} \approx 3.3 \times 10^{-11}\right){ }^{4.5}$ Earlier authors used a factor of 2 instead of $3 / 2$. Measurements at $532 \mathrm{~nm}$ with our $31 \mathrm{~m}$ multi-mode 50 and 100 $\mu \mathrm{m}$ fibers yield $\mathrm{g} \approx 3.8 \times 10^{-11} \mathrm{~m} / \mathrm{W}$ with a $10 \%$ uncertainty. Although our laser output is polarized, injected light is depolarized after a meter or so of transmission through multimode fiber. The SBS reflected-light oscillations of Fig. 5 show a 300 ns period, which is twice the light transit time in the silica fiber.

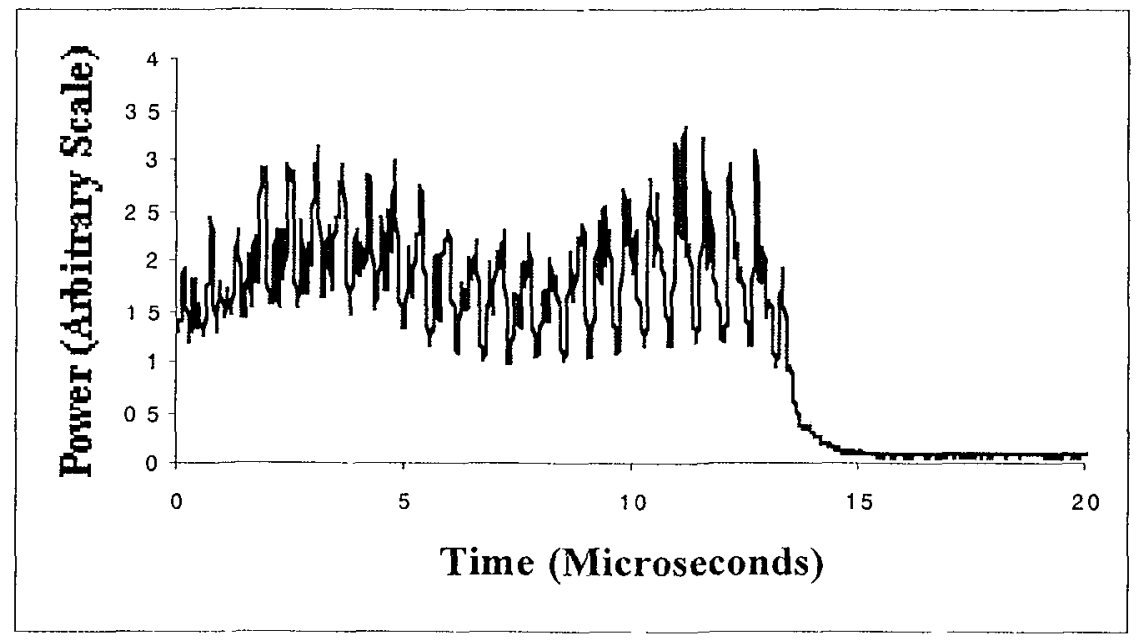

Figure 5: Light reflected by SBS. 


\section{Acknowledgement}

This work was performed under the auspices of the U.S. Department of Energy by the Lawrence Livermore National Laboratory under contract W-7405-Eng-48.

\section{Appendix}

For a Gaussian beam, the distance $z_{2}$ between an injection lens and its waist is given by the following:

$$
\mathrm{Z}_{2}=\mathrm{F}_{\mathrm{a}}+\frac{\left(\mathrm{Zl}-\mathrm{F}_{\mathrm{a}}\right) \mathrm{F}_{\mathrm{a}}^{2}}{\left(\mathrm{Zl}_{1}-\mathrm{F}_{\mathrm{a}}\right)^{2}+\mathrm{ZR}_{\mathrm{R}}^{2}}
$$

where $z_{1}$ is the distance from the upstream waist to the injection lens and $Z_{R 1}$ is the Rayleigh range of the incoming beam. ${ }^{6}$

$$
\mathrm{Z}_{\mathrm{R} 1}=\frac{\pi \mathrm{D}_{01}^{2}}{4 \lambda \mathrm{M}^{2}}
$$

For minimum injected beam size, $R_{a}$ is set equal to $z_{2}$. The diameter $D_{02}$ of the downstream waist is connected to the upstream waist diameter Dol by the following:

$$
D_{02}= \pm \frac{D_{01} F_{a}}{\sqrt{\left(Z_{1}-F_{a}\right)^{2}+Z_{R}{ }^{2}}} \text {. }
$$

\section{References}

${ }^{1}$ C. F. McMillan, et al., "Velocimetry of fast surfaces using Fabry-Perot interferometry", Rev. Sci. Instrum. 29, pp. $1-20$ (1988).

${ }^{2}$ A. E. Siegman and S. W. Townsend, "Output Beam Propagation and Beam Quality from a Multimode Stable-Cavity Laser", IEEE Journal of Quantum Electronics 29, pp. 1212-1217 (1993); T. F. Johnston, Jr., " $\mathrm{M}^{2}$ concept characterizes beam quality", Laser Focus World, May (1990).

${ }^{3}$ D. R. Goosman, "Formulas for Fabry-Perot velocimeter performance using both stripe and multifrequency techniques", 30, pp. 3907-3923 (1991).

${ }^{4}$ Govind Agrawal in Nonlinear Fiber Optics, chapter 9, Second edition, Academic Press (1995).

${ }^{5}$ Walter Koechner, Solid-State Laser Engineering, chapter 10, Fourth Edition, Springer (1996).

${ }^{6}$ S. S. Charschan, Lasers in Industry, p. 141, Van Nostrand Reinhold (1972). 


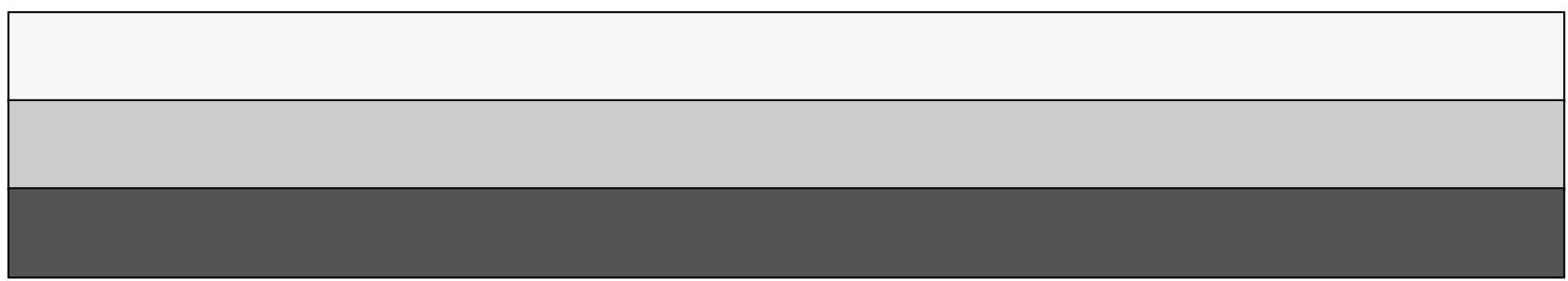

